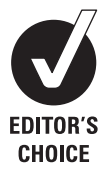

CHOICE

\title{
Unexpected collapse in apparently healthy newborns - a prospective national study of a missing cohort of neonatal deaths and near-death events
}

\author{
Julie-Clare Becher, ${ }^{1}$ Shetty S Bhushan, ${ }^{2}$ Andrew J Lyon'
}

\begin{abstract}
1Department of Neonatology, Simpson Centre for Reproductive Health, Royal Infirmary of Edinburgh, Edinburgh, UK

${ }^{2}$ Neonatal Unit, Ninewells Hospital, Dundee, UK

\section{Correspondence to Dr Julie-Clare Becher, Consultant Neonatologist, Department of Neonatology, Simpson Centre for Reproductive Health, Royal Infirmary of Edinburgh, Little France Crescent, Edinburgh EH16 4SA, UK; julie-clare.becher@luht.scot. nhs.uk}

Accepted 13 May 2011 Published Online First 28 June 2011

\begin{abstract}
Background Sudden and unexpected postnatal collapse (SUPC) of a healthy newborn infant is a rare event, which carries a high risk of mortality and significant neurodisability in survivors. An underlying condition can be found in $60 \%$ of cases who undergo detailed postmortem but in the remainder there are important associations with prone position, breast feeding and primiparous status. The authors undertook a prospective study to ascertain the population incidence of SUPC in the UK.
\end{abstract}

Methods Cases were referred through the British Paediatric Surveillance Unit reporting scheme over a 13-month period. Infants were at $\geq 37$ weeks of gestation, had an Apgar score of $\geq 8$ at 5 min, collapsed within $12 \mathrm{~h}$ in hospital requiring positive pressure ventilation and either died or received ongoing intensive care. Data were collected on maternal and infant characteristics, clinical investigations and 1-year outcome.

Findings 45 cases were reported, an incidence of 0.05/1000 live births of whom 12 infants died. In $15 / 45$ infants, an underlying disease/abnormality was determined. In 30/45 cases (0.035/1000 live births), no such cause was found, but in 24, the clinical/ pathological diagnosis was airway obstruction during breast feeding or in prone position. Mothers were commonly primiparous and unattended by clinical staff before collapse was recognised. Approach to investigation was highly disparate and frequently very limited. Of the 30 infants with no underlying disease/ abnormality, $22(73 \%)$ developed a postasphyxial encephalopathy and 10 had a poor outcome $(33 \%)-5$ died and 5 had neurological sequelae at 1 year.

Interpretation SUPC is rare in any one centre and there is no standard approach to investigation. In those cases where collapse is not due to an underlying abnormality, breast feeding and prone position are important associations. Guidelines for safe postnatal care of infants should include appropriate vigilance of infants particularly where mothers are primiparous or where ability to assess the baby may be impaired.

\section{INTRODUCTION}

Newborn term infants, who are or who appear to be healthy at birth and expected to be at low risk of deterioration, routinely spend their first hours in the care of their mother experiencing 'skin-toskin' and initiating breast feeding. The sudden and unexpected collapse of such an infant is rare but well recognised. ${ }^{1-4}$ There are known associations with primiparous status and postnatal care practices such as breast feeding, prone position and

\section{What is already known on this topic}

- Reports of sudden and unexpected postnatal collapse (SUPC) of a healthy newborn infant are rare.

- Such infants frequently die or survive with neurological sequelae.

- Case series report an association with prone position and breast feeding.

\section{What this study adds}

- The UK population incidence of SUPC within $12 \mathrm{~h}$ is $0.05 / 1000$ term live births and a quarter of infants die.

- One third of infants have an underlying pathological or clinical condition but over half collapse following accidental suffocation, which frequently goes unrecognised by parents.

- Situational risk factors for conventional SUDI are common and routine postnatal practices should be supported with education about signs of infant well-being.

skin-to-skin 356 (figure 1). Despite initial resuscitation, there is a high risk of death or long-term neurological disability. ${ }^{36-8}$ In those infants dying unexpectedly within the first week of life, almost $60 \%$ are explained following detailed perinatal postmortem. ${ }^{9}$

These infants, who are currently unrecognised in national statistics, represent a missing group of child mortality and morbidity due to their rarity in any one region and the difficulty in classification at the time of death.

We sought to establish the population incidence of sudden unexpected postnatal collapse (SUPC) in apparently healthy infants within the UK to describe situational factors and the cause for collapse was determined.

\section{METHODS}

Cases were referred through the British Paediatric Surveillance Unit (BPSU) reporting scheme over a 13-month period between 1 November 2008 and 30 November 2009. The BPSU undertakes active surveillance of uncommon childhood conditions using a network of 3000 multispecialty paediatricians in the UK and Ireland. This list includes 
all hospital, university and community consultant paediatrician members of the Royal College of Paediatrics and Child Health and the Faculty of Paediatrics of the Royal College of Physicians of Ireland. Other respondents include non-College members of some specialty groups such as paediatric pathologists. Participants are sent a monthly case report card containing a 'menu' of conditions under surveillance and are asked to report any new cases or 'none seen'. The overall response rate is over $90 \%$. Positive returns are notified to the study team who then collect further information from clinicians.

Included in the case definition were infants who were at $\geq 37$ weeks of gestation; had an Apgar score of $\geq 8$ at 5 min; collapsed within $12 \mathrm{~h}$ of birth in hospital and required resusci-

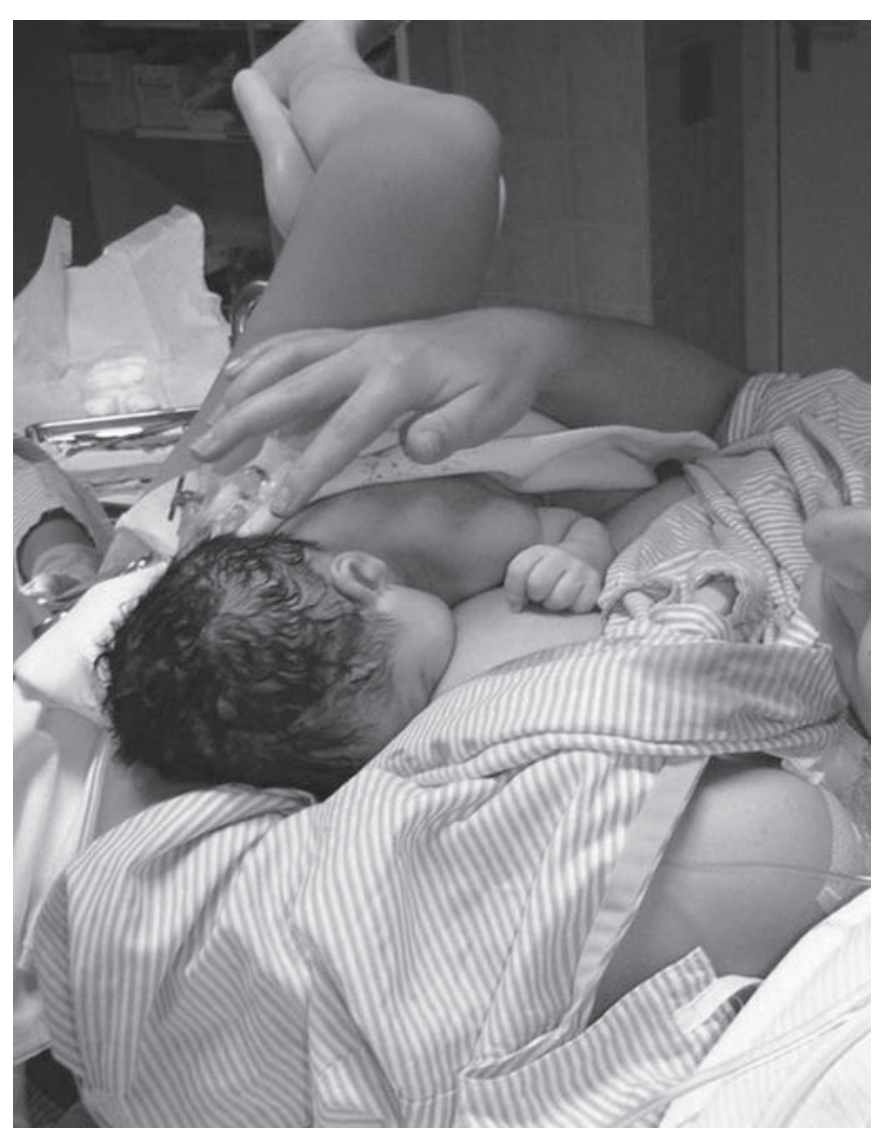

Figure 1 Unrecognised postnatal collapse. Reprinted with courtesy of the Swiss Society of Neonatology and with written permission of parents. Photograph taken by the father. tation with positive pressure ventilation; and died or received ongoing intensive care.

Data were collected on maternal and infant characteristics. Clinical investigations and outcome at discharge and at 1 year of age were recorded.

The study was approved by the National Research and Ethics Service (London REC Ref: 08/H0718/47) and was granted PIAG Section 251 support (Ref: PIAG 5-06 (FT1)/2008). As all data were anonymous for the purposes of analysis, the study did not require written parental consent.

\section{RESULTS}

Ninety-one cases were referred of which 32 were errors and 10 were duplications. There were four further cases that were excluded as eligibility could not be reliably ascertained due to missing data. Forty-five cases of SUPC were reported, which fulfilled the study definition ( $96 \%$ ascertainment). Twelve infants died (27\%) and 33 infants survived to discharge, all of whom had outcome data available.

Table 1 details the cause for collapse in all infants as determined by a pathologist or clinician. Fifteen infants were found to have an underlying condition responsible for their deterioration and of these, six died. In the remaining 30 cases, no underlying condition was found. Of these, there was a clinical or postmortem diagnosis of apparent accidental suffocation in 24 infants, 5 of whom died. In a further 6 cases, no cause was determined and 1 died.

There were 858466 term live births in the UK and Ireland during the 13 months of study. The population incidence of sudden unexpected collapse within the first $12 \mathrm{~h}$ of life was $0.05 / 1000$ $(1 / 19000)$ term live births and the mortality was $0.01 / 1000$ $(1 / 72000)$ term live births. Where no underlying disease/abnormality of the infant was determined $(n=30)$, the population incidence was $0.035 / 1000(1 / 29000)$ term live births.

\section{CHARACTERISTICS OF MOTHER, LABOUR AND DELIVERY Infants with an underlying condition $(n=15)$}

Ten $(67 \%)$ mothers were primiparous. No mothers smoked and all were in good health during pregnancy. Six infants were born by spontaneous vertex delivery, three by instrumental vaginal delivery and six by caesarean section. In six, there was meconium staining of the liquor.

\section{Infants without an underlying condition $(\mathbf{n}=\mathbf{3 0})$}

Twenty-three (77\%) mothers were primiparous. All mothers were in good health prior to pregnancy and two smoked

Table 1 Specified causes of collapse

\begin{tabular}{|c|c|c|c|}
\hline No underlying disease/abnormality determined & 30 infants & Died & $\begin{array}{l}\text { Abnormal neurological } \\
\text { examination by } 1 \text { year }\end{array}$ \\
\hline Apparent accidental suffocation during breast feeding or skin-to-skin & $24(80 \%)$ & 5 & 5 \\
\hline No cause identified & $6(20 \%)$ & 1 & 0 \\
\hline Disease or abnormality & 15 infants & Died & $\begin{array}{l}\text { Abnormal neurological } \\
\text { examination by } 1 \text { year }\end{array}$ \\
\hline Bacterial pneumonia/sepsis ( 2 Group B streptococcus, 3 culture negative) & 5 & 2 & 1 \\
\hline Cardiac (transposition of the great arteries, hypoplastic left heart syndrome) & 2 & 2 & 0 \\
\hline Metabolic disorder (Zellweger's syndrome, unidentified disorder) & 2 & 1 & 1 \\
\hline Intracranial haemorrhage/infarction & 2 & 0 & 1 \\
\hline Meconium aspiration syndrome & 2 & 0 & 0 \\
\hline Severe chronic anaemia (parvovirus) & 1 & 1 & 0 \\
\hline Congenital diaphragmatic hernia & 1 & 0 & 0 \\
\hline
\end{tabular}


tobacco. Four had hypertension in pregnancy. Eight (27\%) mothers were health professionals or ancillary health workers.

Sixteen infants were born by spontaneous vertex delivery, five by instrumental vaginal delivery and nine by caesarean section, one of whom was delivered electively because of a previous caesarean section. Paediatric staff were present at 13 deliveries and in 4, a brief period of intermittent positive pressure ventilation was required. In four cases, there was meconium staining of the liquor. In 11 cases, there was record of a cord gas, median pH 7.18 (range 7.09-7.3) and base deficit $5.3 \mathrm{mmol} / 1$ (range $2-9.5 \mathrm{mmol} / \mathrm{l}$ ).

\section{CHARACTERISTICS OF THE INFANT}

There were 22 (49\%) male infants. The median gestational age was 40 weeks (37-42 weeks) and the mean birth weight was $3328 \mathrm{~g}$ (range 2260-4030 g). All infants (including those given positive pressure ventilation) had Apgar scores of 8 or above at 5 min of age and were assigned to routine postnatal care with their mother.

\section{CIRCUMSTANCES OF COLLAPSE}

The circumstances of collapse are shown in table 2 . The median age at collapse was $70 \mathrm{~min}$ in those with no specific cause for collapse compared with 195 min for those with an underlying condition. In one case, an acute deterioration was witnessed at 6 min of age in an infant who had been vigorous immediately beforehand. This infant died later from refractory hypoxaemia and no cause was determined postmortem.

Collapse occurred between 21:00 and 08:59 h in $25(56 \%)$ cases. Thirteen (29\%) infants were presumed to be feeding at the time of collapse. Thirty $(67 \%)$ women had received analgesia in the form of opiates or had had a regional or general (one case) anaesthetic in the $8 \mathrm{~h}$ preceding the collapse.

In 23 cases, the mother or both parents were unattended with their new baby at the time of collapse. When mothers were left alone with their baby, they recognised signs of collapse in around a third of instances. When another parent was present, they recognised the signs of collapse more often (75\%). Where clinical staff were present in the room, the staff were always first to recognise the infant's deterioration. Only one of eight mothers employed in the health profession recognised signs of deterioration in their infant.
All infants required positive pressure ventilation following recognition of collapse and 22 received cardiac compressions of whom 10 also received resuscitation drugs. Two infants died immediately despite resuscitative efforts (one with sepsis and one with transposition of the great arteries) and 43 infants were subsequently admitted to a neonatal unit.

\section{NEONATAL DETAILS}

Following admission to the neonatal unit, the median $\mathrm{pH}$ within $1 \mathrm{~h}$ of admission was 6.98 (IOR 6.8, 7.18) and median base deficit was $17.7 \mathrm{mmol} / \mathrm{l}$ (IOR 11.4-23.5). Twenty-three infants developed multiorgan dysfunction (53\%) and a further 17 had single organ dysfunction (40\%).

\section{Subgroup of infants without an underlying condition}

Figure 2 shows the range of investigations undertaken to determine the cause of collapse in those infants where an underlying abnormality could not be found. Twenty-two (73\%) of these infants developed a postasphyxial encephalopathy (8 mild, 6 moderate and 8 severe). Ten MRI scans were reported as abnormal: findings included evidence of intracranial haemorrhage, ${ }^{3}$ high signal abnormalities of the white matter ${ }^{6}$ and basal ganglia injury. ${ }^{3}$ One infant had unspecified abnormalities that were described as consistent with an asphyxial insult.

Although not a prespecified data field, three infants were notified as receiving therapeutic hypothermia following collapse and one of them died.

\section{OUTCOME}

Overall, 12 (27\%) of the 45 infants died and 33 infants survived to discharge. Ten of the 12 deaths underwent postmortem examination and two did not (one infant was diagnosed on echocardiogram before death as having hypoplastic left heart syndrome, the other collapsed following presumed accidental suffocation and there was no consent for postmortem). Of the 10 infants undergoing postmortem, an underlying diagnosis was found in 5 (50\%). Table 1 details the cause for collapse in all infants as determined by a pathologist or clinician.

Of the 33 infants who survived to discharge, 8 had neurological abnormalities at 1-year follow-up (24\%). Three of these children had an underlying cause for collapse including Zellweger's syndrome, cerebral infarction and culture-negative sepsis.

Table 2 Circumstances of collapse

\begin{tabular}{lll}
\hline Details of collapse & $\begin{array}{l}\text { Underlying } \\
\text { condition (15) }\end{array}$ & $\begin{array}{l}\text { No underlying } \\
\text { condition (30) }\end{array}$ \\
\hline Age in minutes (median (range)) & $195(10-688)$ & $70(6-643)$ \\
Collapse in first $2 \mathrm{~h}$ & $7(47 \%)$ & $22(73 \%)$ \\
Baby in care of mother/parents at collapse & 13 & 24 \\
Mother alone in room at time of collapse & 7 & 10 \\
$\quad$ Mother recognised baby unwell & $3(43 \%)$ & $2(20 \%)$ \\
Parents alone in room at time of collapse & 1 & 7 \\
$\quad$ Parents recognised baby unwell & $1(100 \%)$ & $5(71 \%)$ \\
Clinical staff in room at time of collapse & 7 & 13 \\
$\quad$ Staff first to recognise baby unwell & $7(100 \%)$ & $13(100 \%)$ \\
Position of baby & & $18(60 \%)$ \\
Mother's breast/chest/abdomen (prone or side) & $3(20 \%)$ & $9(30 \%)$ \\
Arms & $3(20 \%)$ & $3(10 \%)$ \\
In cot (1 prone) & $6(40 \%)$ & 0 \\
Uncertain position & $3(20 \%)$ & \\
\hline
\end{tabular}




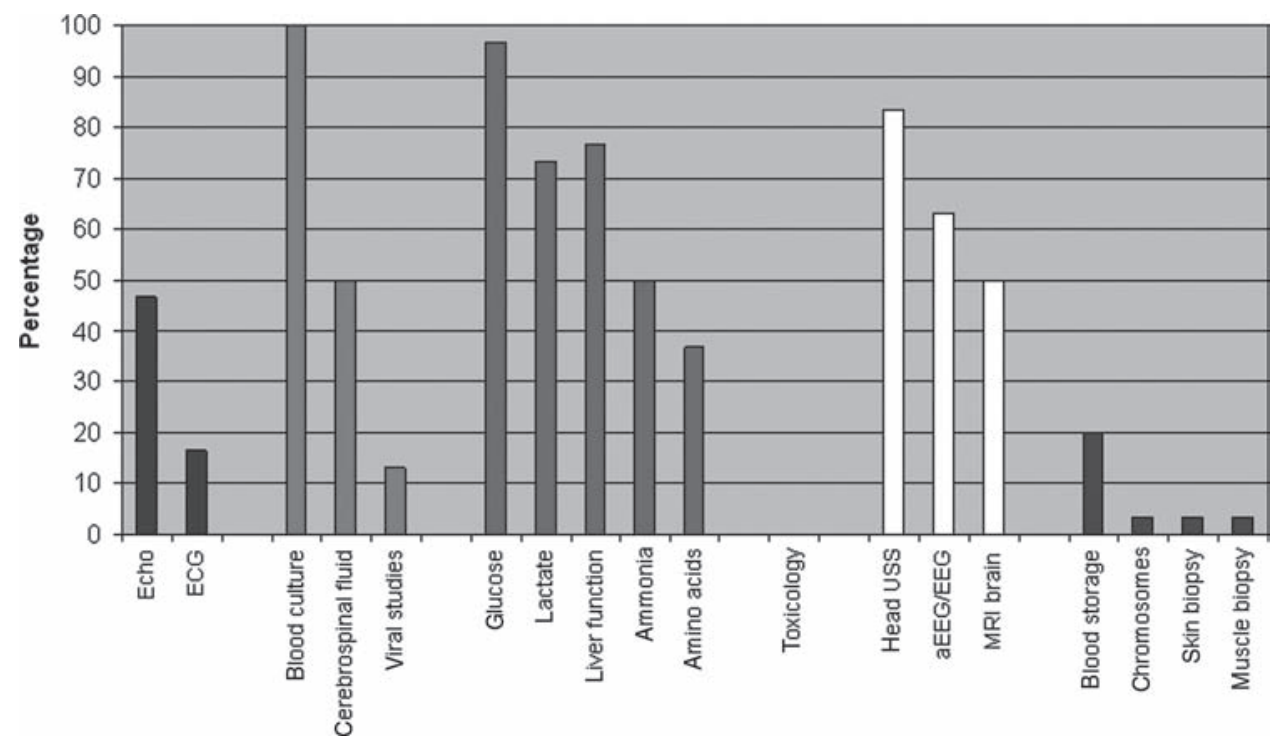

Figure 2 Proportion of infants with no specified cause for collapse undergoing investigation $(n=30)$. USS, ultrasound scan.

\section{Subgroup of infants without an underlying condition}

Nineteen of the 24 infants with presumed accidental suffocation survived to discharge. At 1 year, five were noted to have neurological abnormalities (26\%). Three have cerebral palsy, one has probable cerebral palsy with significant motor delay and the other has mild global delay and hypotonia. All six infants who had no identified cause for collapse were normal at follow-up.

\section{DISCUSSION}

This study is the first to document the population incidence in the UK of SUPC in infants who were assessed as being healthy at birth. The incidence $(0.05 / 1000$ term live births) is consistent with case, regional and national series, suggesting an incidence ranging from between $0.026 / 1000$ live births in the first $24 \mathrm{~h}$ of birth $^{10}$ to $0.27 / 1000$ where collapse occurs within 3 days of birth. ${ }^{2}$ The overall BPSU respondent rate (94.6\% for 2008) is higher than any equivalent UK scheme and ranks highly against other national paediatric surveillance units. However, complete ascertainment of all cases of SUPC occurring within the study period could not be verified. This was due to the complexity of the case definition and the heterogeneity that exists in the classification of such infant deaths, which precluded cross-checking against national and regional databases. As such, the incidence in this study may be an underestimate.

One third of infants had an underlying condition leading to collapse and a further $53 \%$ had situational factors that led to a diagnosis of presumed accidental suffocation. Of the latter infants, over $40 \%$ had a poor outcome of death or disability by 1 year. The association of skin-to-skin, breast feeding and prone position in SUPC has been reported previously. ${ }^{3-5}$ Skinto-skin practice involves placing a baby naked and prone on the mother's chest and covered with a warm blanket. In a recent meta-analysis, skin-to-skin was associated with increased duration of breast feeding and maternal bonding behaviour without serious adverse events. ${ }^{11}$ The benefits of breast feeding are undisputed. Both early skin-to-skin and breast feeding are promoted increasingly in maternity hospitals around the UK and are often accompanied by withdrawal of clinical staff from the maternity room so as to facilitate uninterrupted bonding.
The prevention of sudden infant death syndrome is promoted in parental information at discharge from hospital and in health centres throughout the UK. Parents are explicitly warned of the dangers of placing their infant prone to sleep, allowing blankets to cover their infant's head or putting to sleep on a surface which is not firm and flat. Co-bedding is actively discouraged particularly when the mother may have taken sedative drugs or is unusually tired to a point at which she would find it difficult to respond to her baby. ${ }^{12} 13$

The high incidence of skin-to-skin, prone position and breast feeding at the time of collapse and the frequency of maternal sedation following an exhausting labour would suggest that risk factors for conventional sudden infant death syndrome are as important in the immediate newborn period as they are on discharge from hospital. Indeed it is artificial to draw a distinction because an infant is on hospital premises, particularly where there is a lack of clinical supervision. Moreover, the proximity to delivery in such infants suggests that during this critical period of adaptation, newborn infants may be less able to cope with hypoxic stress, such as airway obstruction. ${ }^{10}$

The finding that the majority of mothers were primiparous (compared with around $50 \%$ of all deliveries) is consistent with the literature on SUPC 35814 as is the finding that new parents were often left alone with their newborn infant. ${ }^{6} 1516$ Even where an underlying condition was responsible for the infant's collapse, the fact that mothers rarely recognised their infant's deterioration suggests that inexperience and a lack of knowledge of the signs of wellness are important factors and such delay in recognition may contribute to the poor outcomes observed. Vital signs such as colour or respiratory effort may also be more difficult to observe if maternal mobility is impaired due to pain, postoperative status or spinal anaesthesia.

For the significant majority of newborn infants, it is clear that breast feeding and skin-to-skin practices are safe, beneficial and should be recommended. For such recommendations to be safe for all infants, guidelines should include appropriate vigilance of infants. Responsibility should be with clinical staff to ensure that during breast feeding and skin-to-skin practices, parents are able to assess the airway, breathing and colour of their infant. Where parental observation of these parameters may be impaired by exhaustion or sedation, the responsibility for vigilance should reside with the clinical staff. 
This study established that the approach to investigation of these infants by clinicians was highly disparate with few cases undergoing systematic investigation. Where death occurs, postmortem and referral to the Coroner is not universal despite the fact that these are sudden and unexpected deaths in hospital and that pathological investigation has higher diagnostic yield in infants dying in the first week of life compared with later SUDI (Sudden Unexpected Death in Infancy). ${ }^{17}$ Professional guidance for the investigation of sudden unexpected death in infancy exists ${ }^{18}$ but has little diagnostic emphasis on the immediate newborn period where structural anomalies and metabolic conditions are more likely. ${ }^{9}$ In addition, many clinicians fail to recognise these infants as SUDI as many undergo resuscitation at the time of collapse and die later from hypoxicischaemic consequences. Around $50-80 \%$ of babies survive following SUPC and investigation of survivors is not covered by existing protocols. ${ }^{12}$ Recently developed national guidelines specific to infants who collapse suddenly in the first week of life should improve diagnostic certainty as well as clarify prognosis and ongoing management in those who survive. ${ }^{19}$

Contributors J-CB: literature search, study design, grant application, data collection, data analysis and interpretation, and writing. AJL: literature search, study design, grant application, data analysis and interpretation, and writing. SSB: data collection.

Acknowledgements The authors acknowledge the BPSU, supported by the Department of Health, for facilitating the data collection, and the reporting clinicians, particularly those who completed the questionnaires. Any views expressed in this publication are those of the investigator and not necessarily those of the BPSU or DH.

Funding The study was funded by WellChild from whom all authors are independent.

Competing interests (1) J-CB, SSB and AJL have support from WellChild for the submitted work; (2) J-CB, SSB and AJL have no relationships with any company that might have an interest in the submitted work in the previous 3 years; (3) their spouses, partners, or children have no financial relationships that may be relevant to the submitted work and (4) J-CB, SSB and AJL have no non-financial interests that may be relevant to the submitted work.

Patient consent Obtained.

Ethics approval This study was conducted with the approval of the National Research and Ethics Service (London REC Ref: 08/H0718/47).

Provenance and peer review Not commissioned; externally peer reviewed.

\section{REFERENCES}

1. Polberger S, Svenningsen NW. Early neonatal sudden infant death and near death of fullterm infants in maternity wards. Acta Paediatr Scand 1985;74:861-6.
2. Rodríguez-Alarcón J, Melchor JC, Linares A, et al. Early neonatal sudden death or near death syndrome. An epidemiological study of 29 cases. Acta Paediatr 1994;83:704-8.

3. Hays $\mathbf{S}$, Feit $P$, Barré $P$, et al. [Respiratory arrest in the delivery room while lying in the prone position on the mothers' chest in 11 full term healthy neonates] Arch Pediatr 2006;13:1067-8.

4. Branger B, Savagner C, Roze JC, et al.; Pédiatres des Maternités des Pays-de-la-Loire. [Eleven cases of early neonatal sudden death ou near death of full term and healthy neonates in maternity wards]. J Gynecol Obstet Biol Reprod (Paris) 2007;36:671-9.

5. Gatti $\mathbf{H}$, Castel $\mathrm{C}$, Andrini $\mathrm{P}$, et al. [Cardiorespiratory arrest in full term newborn infants: six case reports]. Arch Pediatr 2004;11:432-5.

6. Toker-Maimon 0, Joseph LJ, Bromiker R, et al. Neonatal cardiopulmonary arrest in the delivery room. Pediatrics 2006;118:847-8.

7. Burchfield DJ, Rawlings DJ. Sudden deaths and apparent life-threatening events in hospitalized neonates presumed to be healthy. Am J Dis Child 1991; 145:1319-22

8. Peters C, Becher JC, Lyon AJ, et al. Who is blaming the baby? Arch Dis Child Fetal Neonatal Ed 2009;94:F377-8.

9. Weber MA, Ashworth MT, Risdon RA, et al. Sudden unexpected neonatal death in the first week of life: autopsy findings from a specialist centre. J Matern Fetal Neonatal Med 2009;22:398-404.

10. Poets A, Steinfeldt R, Poets CF. Sudden deaths and severe apparent life-threatening events in term infants within 24 hours of birth. Pediatrics 2011;127:e869-73.

11. Moore ER, Anderson GC, Bergman N. Early skin-to-skin contact for mothers and their healthy newborn infants. Cochrane Database Syst Rev 2007;3:CD003519.

12. Department of Health. Reduce the Risk of Cot Death. 2009. http://www.dh.gov.uk/ prod consum dh/groups/dh digitalassets/documents/digitalasset/dh 096299.pdf (accessed 17 Jun 2011)

13. UK Baby Friendly Initiative and the Foundation for the Study of Infant Deaths. Sharing a Bed with Your Baby. A Guide for Breastfeeding Mothers. London: UNICEF, 2008. http://www.unicef.org.uk/Documents/Baby_Friendly/Leaflets/3/ sharingbedleaflet.pdf (accessed 17 Jun 2011).

14. Foran A, Cinnante C, Groves A, et al. Patterns of brain injury and outcome in term neonates presenting with postnatal collapse. Arch Dis Child Fetal Neonatal Ed 2009;94:F168-77.

15. Dageville C, Pignol J, De Smet S. Very early neonatal apparent life-threatening events and sudden unexpected deaths: incidence and risk factors. Acta Paediatr 2008;97:866-9.

16. Espagne $\mathbf{S}$, Hamon I, Thiébaugeorges 0 , et al. [Sudden death of neonates in the delivery room]. Arch Pediatr 2004;11:436-9.

17. Weber MA, Ashworth MT, Risdon RA, et al. The role of post-mortem investigations in determining the cause of sudden unexpected death in infancy. Arch Dis Child 2008:93:1048-53.

18. Royal College of Pathologists. Sudden Unexpected Death in Infancy: A Multi-Agency Protocol for Care and Investigation. London: Royal College of Pathologists, 2004

19. WellChild. Guidelines for the Investigation of Newborn Infants Who Suffer a Sudden and Unexpected Postnatal Collapse in the First Week of Life: Recommendations from a Professional Group on Sudden Unexpected Postnatal Collapse. London, 2011. http://www.bapm.org/media/documents/SUPC\%20 Booklet.pdf (accessed 17 Jun 2011). 